\title{
Long term recurrence free survival in a stage IV gallbladder cancer treated with chemotherapy plus trastuzumab and salvage liver resection
}

\author{
Mikel Prieto ${ }^{1,2}$, Mikel Gastaca ${ }^{1,2}$, Patricia Ruiz ${ }^{1}$, Alberto Ventoso', \\ Ibone Palomares ${ }^{1}$, Arkaitz Perfecto ${ }^{3}$, and Andrés Valdivieso ${ }^{1,2}$
${ }^{1}$ Hepatobiliary Surgery and Liver Transplantation Unit, General and Digestive Surgery Department, Cruces University Hospital, ${ }^{2}$ University of the Basque Country, \\ ${ }^{3}$ General and Digestive Surgery Department, Cruces University Hospital, Bilbao, Spain
}

\begin{abstract}
Surgery is the only treatment for biliary tract cancer with long term survival. Unfortunately, most patients are diagnosed at stage IV with distant metastases. In these circumstances, life expectancy is less than one year due to aggressive tumour biology and a lack of effective systemic therapies. HER2 overexpression or amplification is predominantly seen in extrahepatic cholangiocarcinoma and gallbladder cancer (10-18\%) and rarely in intrahepatic cholangiocarcinoma (1\%). Trastuzumab is a monoclonal antibody that targets HER-2. We present a clinical case with a stage IV gallbladder cancer (liver and interaortocaval lymph node metastases), which presented progression during first-line chemotherapy treatment, which prompted a change in therapy to study the Her $2 / \mathrm{Neu}$ mutation which showed an intense positive overexpression. A combination of HER2/Neu-directed therapy (Trastuzumab) with second-line chemotherapy, was able to achieve a long term complete radiological, metabolic, and biochemical response. A curative intention surgery was performed and the patient is alive and recurrence-free at five years. To the best of our knowledge, we present a case which is the first report of a patient with a Stage IV gallbladder cancer who achieved a five-year survival without recurrence after a conversion therapy combining chemotherapy plus Trastuzumab and radical salvage surgery. (Ann Hepatobiliary Pancreat Surg 2019;23:403-407)
\end{abstract}

Key Words: Bile duct cancer; Gallbladder cancer; Conversion surgery; Her-2; Trastuzumab

\section{INTRODUCTION}

Surgery is the only treatment for biliary tract cancer (BTC) that have shown long term survival. BTCs are classified based on their anatomical site in the biliary tree [intrahepatic (IHCC), extrahepatic cholangiocarcinoma (EHCC)], and gallbladder cancer (GBC). Most patients are diagnosed in stage IV with distant metastases ${ }^{1}$ with a life expectancy of less than one year due to aggressive tumour biology and lack of effective systemic therapies. ${ }^{2}$

In cases with non-resectable BTC (locally advanced, recurrent, or metastatic), the current standard of care is systemic chemotherapy with gemcitabine and cisplatin. This regimen was established by the ABC-02 trial, the largest randomised phase III study to date, which showed a sur- vival benefit of the combination of gemcitabine and cisplatin opposed to gemcitabine alone ( 11.7 vs. 9 months). ${ }^{2}$ Nevertheless, clinical response rates to these regimens are low, with $<10 \%$ long term survival for all BTC subtypes and a complete response only in exceptional cases. ${ }^{2}$ Other chemotherapy combinations (5-Fluoruracile, capecitabine, oxaliplatin, and irinotecan) have only marginal improvements in survival. ${ }^{3,4}$

BTC has multiple potential mutations that could be used as a target for treatment, but there is a significant lack of evidence of such novel therapeutic strategies. ${ }^{5}$ Due to this, a greater appreciation of the molecular heterogeneity across the BTC subtypes must be paid, realising that these anatomically classified subgroups (IHCC, EHCC, or GBC) exhibit distinct molecular and potential ther-

Received: April 20, 2019; Accepted: July 11, 2019

Corresponding author: Mikel Prieto

Department of Hepatobiliary and Liver Transplant Surgery, Cruces University Hospital, Plaza de Cruces Sn, Bilbao, cp 48903, Spain Tel: +34-689407311, Fax: +34-9466006000, E-mail: mikel.prietocalvo@osakidetza.eus

Copyright (C) 2019 by The Korean Association of Hepato-Biliary-Pancreatic Surgery

This is an Open Access article distributed under the terms of the Creative Commons Attribution Non-Commercial License (http://creativecommons.org/ censes/by-nc/4.0) which permits unrestricted non-commercial use, distribution, and reproduction in any medium, provided the original work is properly cited. Annals of Hepato-Biliary-Pancreatic Surgery • pISSN: 2508-5778 - elSSN: 2508-5859 
apeutic strategies (Fig. 1). ${ }^{6}$ Few cases of successful chemotherapy converting from initially metastatic BTC to resectable BTC have been reported ${ }^{4,7-10}$ and none of them have combined biological therapy with long-term and disease-free survival. There is no current general consensus regarding this issue.

\section{CASE}

We present the case of a 44-year-old male suffering from diffuse abdominal pain who was diagnosed with GBC and with liver and distant lymph node metastases

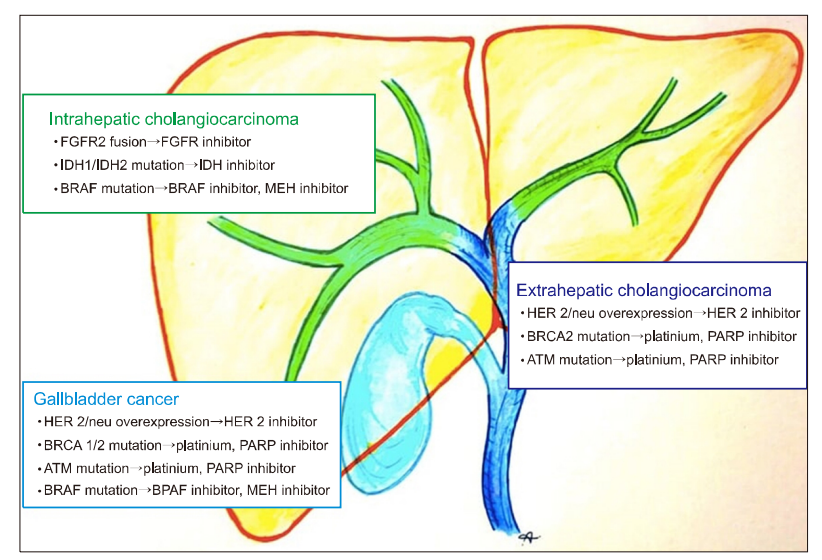

Fig. 1. Potentially genetic alterations and targered therapies. in November 2012 and referred to our centre tumour board. The work-up showed a CA $19-9$ of 22,000 U/ml with no other abnormalities in the laboratory test. The CT, MRI, and PET-CT demonstrated a GBC with liver parenchymal infiltration and multiple liver metastases (Segments IVa-V-VIII) associated with suspected local and distant (interaortocaval) lymph nodes metastases. No vascular or bile duct invasion was detected (Fig. 2). An ultrasound biopsy of the liver metastases was performed which confirmed liver metastases from pancreatobiliary adenocarcinoma origin (CK7+, CK20-, P53+, K-RAS-, CDX2+, and BRAF-) (Fig. 3). From December 2012 to January 2013, three cycles of Gencitabine and Cisplastine-based therapy were started. In spite of this therapy, there was a worsening in the CA 19-9 levels $(63.000 \mathrm{U} / \mathrm{ml})$ and in the radiological findings. Her $2 / \mathrm{Neu}$ mutation was then studied, which showed an intense positive overexpression. Our multidisciplinary team decided to change the chemotherapy to Capecitabine plus Oxaliplatin and Trastuzumab for eight cycles. After six months, a complete radiological (Fig. 2) and metabolic response (PET-CT) (with normalisation in the CA 19-9 levels) was observed (Fig. 4). In November 2013, an exploratory laparotomy was carried out after four months of stable response without treatment. During the surgical procedure, no liver metastases were found on contrast-enhanced ultrasonography. Intraoperative inter-
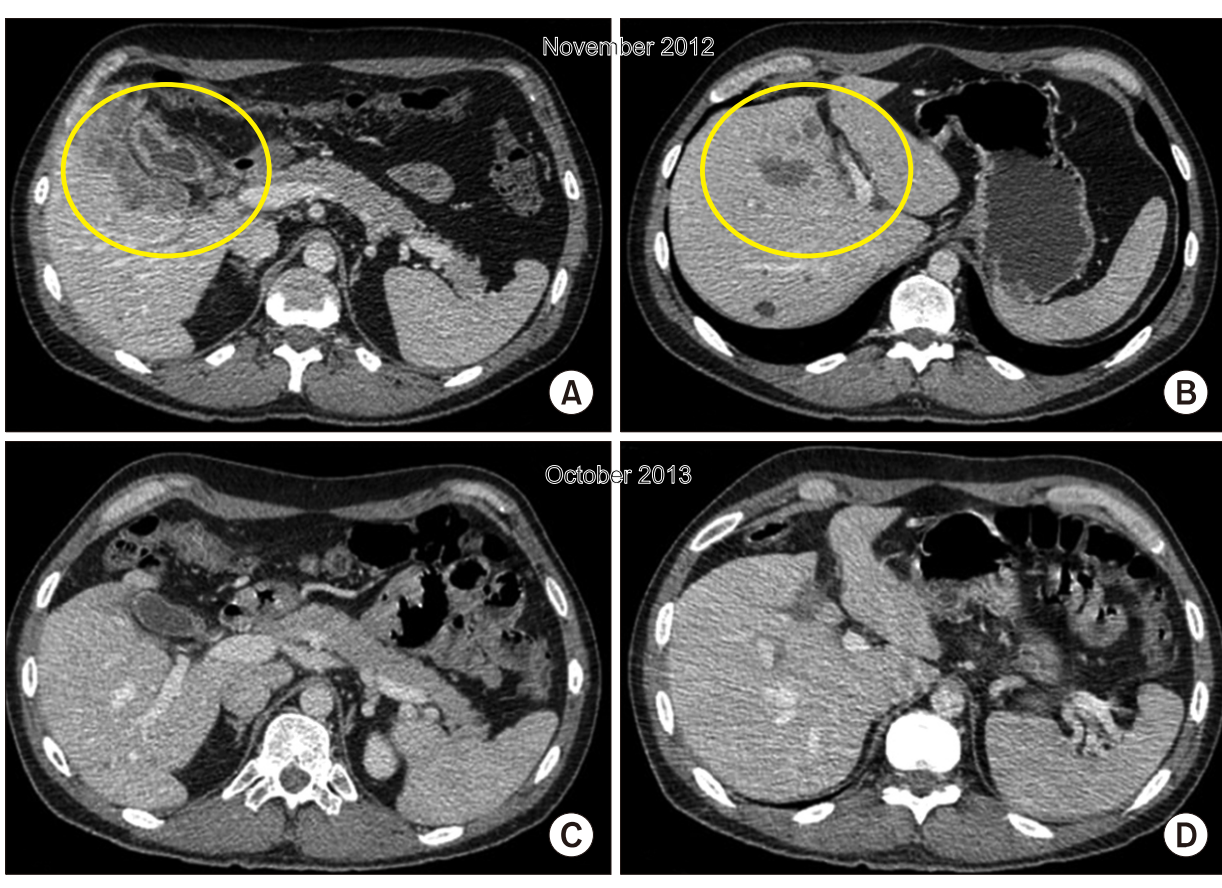

Fig. 2. CT: GBC with liver infiltration, yellow circle, (A) and liver metastases, yellow circle (B). Complete radiological response (C and $\mathrm{D})$. 

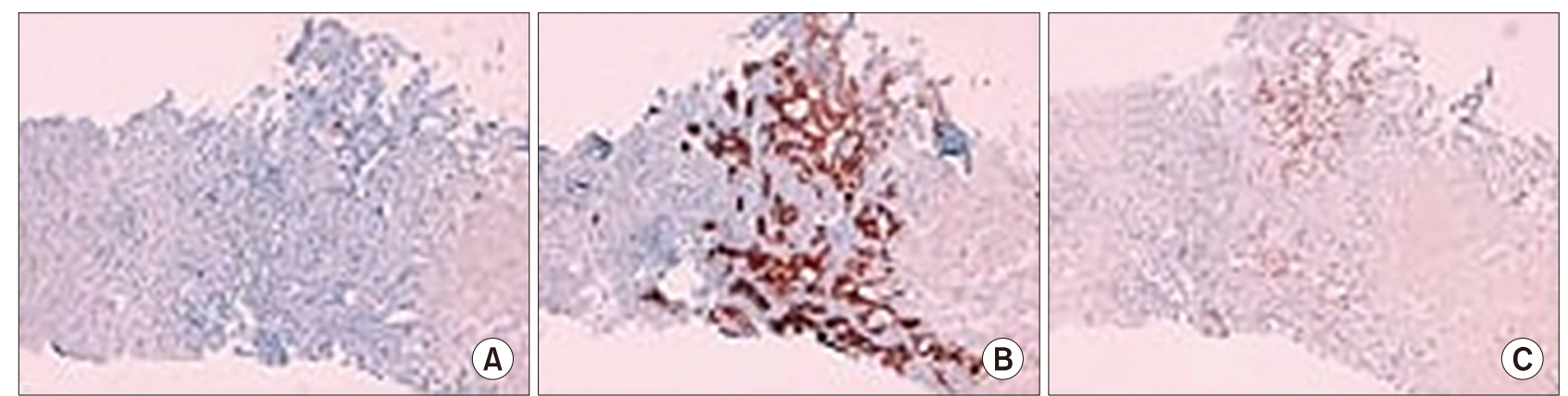

Fig. 3. (A) $\mathrm{CDK} 7+$, (B) $\mathrm{CDK} 20-$, (C) $\mathrm{CDX} 2+$.

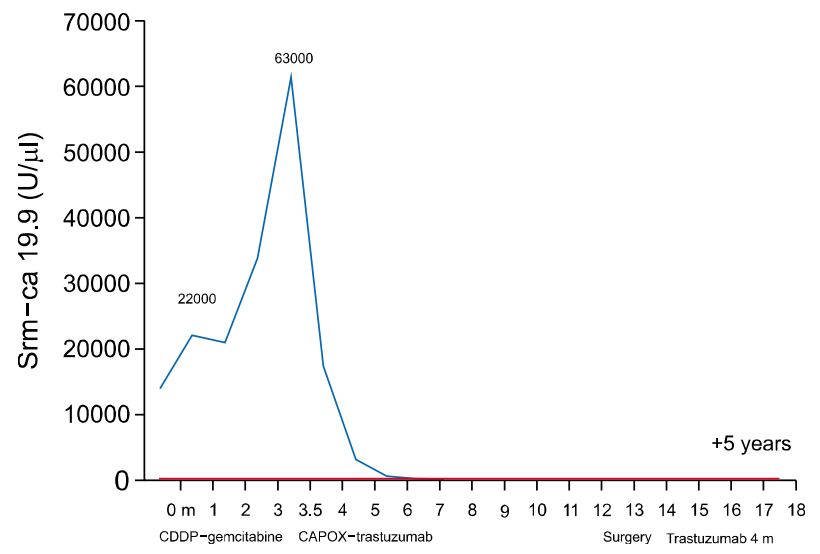

Fig. 4. Biological response.

aortocaval lymph node and cystic duct frozen sections were negative for malignancy. Nevertheless, taking into account the extension of the initial disease, a mesohepatectomy associated with hiliar and interaortocaval lymphadenectomy was performed. The patient developed a Grade IIIa Dindo-Clavien morbidity (biliar fístula) which was managed with an ultrasound-guided puncture. He was discharged on postoperative day 22. Adjuvant therapy with Trastuzumab was administrated for four months. The histopathological exam demonstrated a moderately differentiated gallbladder adenocarcinoma pG2T2N0 (+0/15) M0 (no liver or lymph node metastases), and intense liver multifocal fibrosis. The cystic duct was negative for tumour. Today, the patient is now recurrence-free at five years.

\section{DISCUSSION}

The HER2/neu gene is located on the 17q12-q21 chromosomal region acting as an oncogene in several human cancers. ${ }^{11}$ The HER2 gene is a key driver in the development of several solid tumours, including breast, ovarian, and gastric cancer. Overexpression is observed in approximately $20 \%$ of breast/ovarian cancers and $12 \%$ of gastric cancers. ${ }^{12}$ HER2 overexpression or amplification are predominantly seen in EHCC and GBCs (10-18\%), ${ }^{13}$ and rarely in IHCC (1\%). ${ }^{14}$ The prognostic role of HER2 overexpression or amplification is still controversial in BTC. Some studies failed to demonstrate the prognostic impact of HER2 overexpression, probably due to the relatively small sample size and heterogeneity of the patients studied, ${ }^{14}$ while other reports showed that it could be a bad prognosis factor. ${ }^{15}$

Trastuzumab is a monoclonal antibody that targets HER2. Javle et al. ${ }^{16}$ reported results from a retrospective study with Trastuzumab and concurrent chemotherapy in BTC. HER2 overexpression or amplification was present in eight out of nine GBC and three out of five EHCC. Moreover, the GBC group with HER2 overexpression or amplification experienced radiological stability in three out of eight cases, partial response in four out of eight, and a complete response in one out of eight. On the other hand, none of the EHCC had a radiological response, concluding that HER2/neu-directed therapy is a promising avenue for patients with GBC with gene amplification. ${ }^{16}$ Recently, with the preliminary data from the MyPathway trial, a prospective multicentric phase IIA study, it has been proposed that HER2/neu blockage with Pertuzumab plus Trastuzumab could be a promising treatment strategy for HER2-positive metastatic BTC. In this study, 11 patients with HER2-positive BTC were enrolled. With a median follow-up of 4.2 months, three patients had stability, and four had a partial response for more than four months. ${ }^{17}$ Currently, there is an ongoing Phase II clinical trial (ASLAN001), a pan-HER kinase inhibitor in refractory advanced or metastatic BTC (NCT02609958). 
On the other hand, most of the data for neoadjuvant or conversion chemotherapy followed by surgery comes from small trials and case reports of BTC with advanced unresectable or metastatic disease. ${ }^{4,7-10,18,19}$ None of these studies combined biological therapy with long-term and disease-free survival as the present case. Kato et al. ${ }^{18}$ revealed that the neoadjuvant Gemcitabine regimen is able to downsize tumours towards resectable stages in up to $36.4 \%$ with a comparable survival to those who were resectable at first (45\% vs. $40.8 \%$ survival at five years). Even though the actual standard of care in unresectable BTC is Gemcitabine plus Cisplatin, ${ }^{2}$ a prospective trial comparing Gemcitabine plus Cisplatin versus Gemcitabine alone, failed to demonstrate any differences in resection and survival rates. ${ }^{19}$

Finally, there are no definitive radiological, metabolic, or biochemical criteria to define the adequate timing of possible salvage surgery after neoadjuvant or conversion chemotherapy for unresectable BTC. In our case, we performed a CT scan with CA 19-9 levels every four months during chemotherapy treatment and a PET-CT once there was a complete radiological and biochemical maintained response. Moreover, we think that the assessment of disease stability without treatment prior to surgery as a testtime during a defined period, but not inferior to four months, is crucial in order to re-check the biological aggressiveness of the tumour. Our patient was not initially treated as neoadjuvant but was treated in a palliative setting. Progression during first-line treatment prompted the change to study the Her 2/Neu mutation which showed an intense positive overexpression. Combination of HER2/neu-directed therapy (Trastuzumab) with secondline chemotherapy was able to achieve a long-term complete radiological, metabolic, and biochemical response. A curative intention surgery was performed and the patient is still alive recurrence-free after five years.

Conversion therapy in BTC has recently attracted attention, although there is insufficient evidence regarding the best chemotherapy treatment. To the best of our knowledge, the present case is the first report of a patient with a Stage IV GBC who achieved a five-year survival without recurrence after a conversion therapy combining chemotherapy plus Trastuzumab and radical salvage surgery. Trastuzumab therapy could be a promising treatment option in patients with HER2-positive advanced GBC, but further clinical trials are needed.

\section{REFERENCES}

1. Miyakawa S, Ishihara S, Horiguchi A, Takada T, Miyazaki M, Nagakawa T. Biliary tract cancer treatment: 5,584 results from the Biliary Tract Cancer Statistics Registry from 1998 to 2004 in Japan. J Hepatobiliary Pancreat Surg 2009;16:1-7.

2. Valle J, Wasan H, Palmer DH, Cunningham D, Anthoney A, Maraveyas A, et al.; ABC-02 Trial Investigators. Cisplatin plus gemcitabine versus gemcitabine for biliary tract cancer. N Engl J Med 2010;362:1273-1281.

3. Chong DQ, Zhu AX. The landscape of targeted therapies for cholangiocarcinoma: current status and emerging targets. Oncotarget 2016;7:46750-46767.

4. Sharma A, Mohanti B, Raina V, Shukla N, Pal S, Dwary A, et al. A phase II study of gemcitabine and oxaliplatin (Oxigem) in unresectable gall bladder cancer. Cancer Chemother Pharmacol 2010;65:497-502.

5. Zhao DY, Lim KH. Current biologics for treatment of biliary tract cancers. J Gastrointest Oncol 2017;8:430-440.

6. Morizane C, Ueno M, Ikeda M, Okusaka T, Ishii H, Furuse J. New developments in systemic therapy for advanced biliary tract cancer. Jpn J Clin Oncol 2018;48:703-711.

7. Watanabe T, Furuse J, Okano N, Suzuki Y, Kamma H, Sugiyama M. A pathological complete response after combined chemotherapy of gemcitabine and S-1 in advanced biliary tract cancer with para-aortic lymph nodes metastasis: a case report. Surg Case Rep 2017;3:26.

8. Slupski MW, Szczylik C, Jasinski MK. Unexpected response to systemic chemotherapy in case of primarily nonresectable advanced disseminated intrahepatic cholangiocarcinoma. World $\mathrm{J}$ Surg Oncol 2007;5:36.

9. Oshiro Y, Takahashi K, Sasaki R, Kondo T, Sakashita S, Ohkohchi N. Adjuvant surgery for advanced extrahepatic cholangiocarcinoma. World J Gastroenterol 2013;19:6934-6938.

10. Lim JH, Ryu JK, Choi YJ, Kwon J, Kim JY, Lee YB, et al. A case of common bile duct cancer that completely responded to combination chemotherapy of gemcitabine and TS-1. Gut Liver 2013;7:371-376.

11. Gutierrez C, Schiff R. HER2: biology, detection, and clinical implications. Arch Pathol Lab Med 2011;135:55-62.

12. Yang XR, Chang-Claude J, Goode EL, Couch FJ, Nevanlinna $\mathrm{H}$, Milne RL, et al. Associations of breast cancer risk factors with tumor subtypes: a pooled analysis from the Breast Cancer Association Consortium studies. J Natl Cancer Inst 2011;103: 250-263.

13. Ross JS, Wang K, Thomas Catenacci DV, Chmielecki J, Ail SM, Elvin JA, et al. Comprehensive genomic profiling of biliary tract cancers to reveal tumor-specific differences and genomic alterations. J Clin Oncol 2015;33(3 Suppl):231.

14. Yoshikawa D, Ojima H, Iwasaki M, Hiraoka N, Kosuge T, Kasai $\mathrm{S}$, et al. Clinicopathological and prognostic significance of EGFR, VEGF, and HER2 expression in cholangiocarcinoma. Br J Cancer 2008;98:418-425.

15. Andersen JB, Spee B, Blechacz BR, Avital I, Komuta M, Barbour $A$, et al. Genomic and genetic characterization of cholangiocarcinoma identifies therapeutic targets for tyrosine kinase inhibitors. Gastroenterology 2012;142:1021-1031.e15.

16. Javle M, Churi C, Kang HC, Shroff R, Janku F, Surapaneni R, et al. HER2/neu-directed therapy for biliary tract cancer. J Hematol Oncol 2015;8:58.

17. Javle MM, Hainsworth JD, Swanton C, Burris HA, Kurzrock R, 
Sweeney C, et al. Pertuzumab + trastuzumab for HER2-positive metastatic biliary cancer: preliminary data from MyPathway. J Clin Oncol 2017;35(4 Suppl):402.

18. Kato A, Shimizu H, Ohtsuka M, Yoshidome H, Yoshitomi H, Furukawa K, et al. Surgical resection after downsizing chemotherapy for initially unresectable locally advanced biliary tract cancer: a retrospective single-center study. Ann Surg Oncol 2013;
20:318-324

19. Kato A, Shimizu H, Ohtsuka M, Yoshitomi H, Furukawa K, Takayashiki $\mathrm{T}$, et al. Downsizing chemotherapy for initially unresectable locally advanced biliary tract cancer patients treated with gemcitabine plus cisplatin combination therapy followed by radical surgery. Ann Surg Oncol 2015;22 Suppl 3:S1093-S1099. 